\title{
DYNAMICS OF ROTATIONALLY PERIODIC STRUCTURES
}

\author{
D. L. THOMAS \\ Central Electricity Research Laboratories, Leatherhead, Surrey, England
}

\begin{abstract}
SUMMARY
The paper considers the finite element analysis of the free, undamped and the forced damped vibrations of rotationally periodic structures. Associated with every natural frequency (except for those for which the deflection is the same at corresponding points on every substructure) there are a pair of orthogonal mode shapes, with eigenvectors $\{u\}$ and $\{\tilde{u}\}$. The complex vector $\{z\}=\{u\}+i\{\bar{u}\}$ is also an eigenvector of the equations of motion, and represents a rotating normal mode. The deflection of one substructure has the same amplitude as, and a constant phase difference from, the deflection of the preceding substructure. It is therefore possible to analyse the complete structure by considering only one substructure, and applying appropriate complex constraints at its boundary with the following substructure, so as to impose this phase difference. The method has been implemented in a computer program and is illustrated by analyses of an alternator end winding, a cooling tower with legs, and a wheel of turbine blades.

For forced vibration, it is shown that any arbitrary oscillatory force can be decomposed into a series of rotating forces. For any one of these rotating components, there is a fixed relationship between the amplitude and phase of the force acting on one substructure, and that acting on an adjoining substructure. This relationship, which does not involve any approximation, can be used to enable a series of calculations of the response of one substructure to be performed instead of one on the whole structure. A series of calculations on an individual substructure normally requires much less computer time and storage than a single calculation on the complete structure.
\end{abstract}

\section{INTRODUCTION}

Many engineering structures have a more or less cylindrical form. In some cases, such as certain pipes, chimneys, or pressure vessels, the structures are axisymmetric, and there is now a considerable range of computer programs available for analysing these, taking full advantage of their axisymmetric nature. However, if a structure is not exactly axisymmetric, and cannot be represented approximately by an axisymmetric idealization, it is at present necessary to analyse the whole structure.

The class of structure considered in this paper consists of those which have rotational periodicity. By this is meant that, if the geometry for any radial and axial position is defined at some angle $\theta$, it is identical at $\left(\theta+n \phi_{0}\right)$, where $\phi_{0}$ is $2 \pi / N$, and $n$ and $N$ are integers. $N$ is structure-dependent and $n$ is any integer less than $N$. It follows that once the geometry has been defined over a sector or substructure from $\theta$ to $\theta+\phi_{0}$, the remainder of the structure can be obtained by repeated rotations through $\phi_{0}$. (Note that the sector can start at different $\theta$ positions for different values of $r$ and $z$ ). Structures which possess this property include some alternator end-windings, bladed turbine discs, and cooling towers with legs. These are all complex non-axisymmetric structures for which a complete analysis is impracticable for computer storage and cost reasons. In this paper it is shown how the property of rotational 
periodicity can be exploited to enable a series of analyses on one sector to be used to obtain all the information available from an analysis of the complete assembly.

A cooling tower with legs is an example of a rotationally periodic structure. Figure 1 shows a tower from Didcot Power Station. This has 40 pairs of legs. If the geometry of the tower is completely defined for an angular segment of $2 \pi / 40 \mathrm{rad}$, such as the substructure boundaries

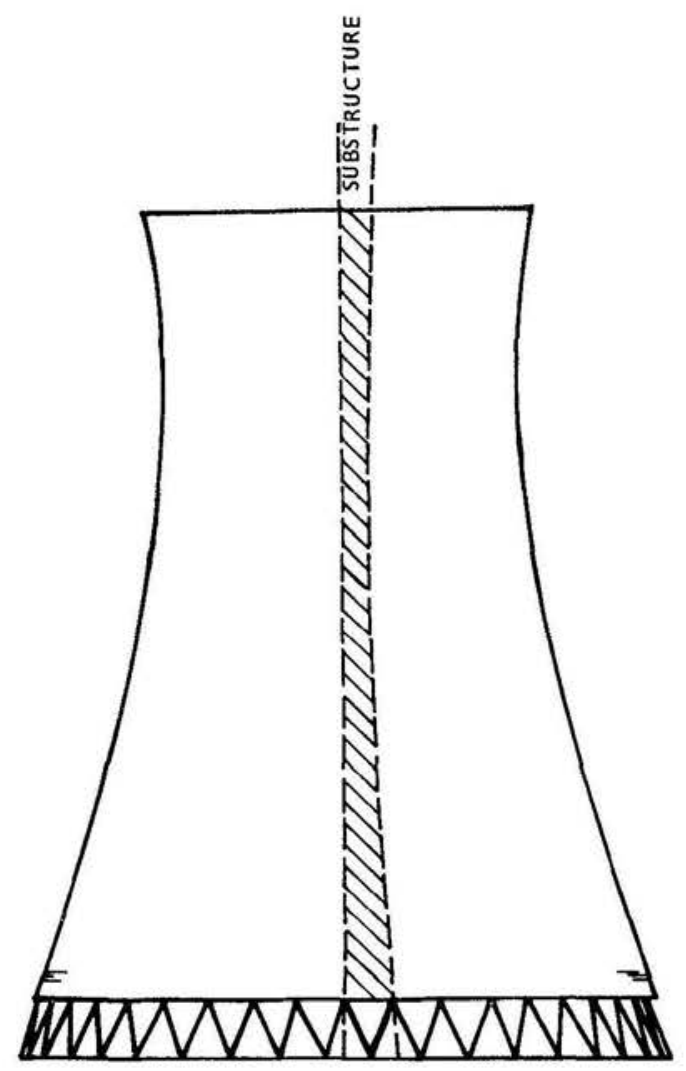

Figure 1. Didcot Cooling Tower, showing substructure

shown in Figure 1, the rest of the tower can be generated by repeated rotations of the segment through $2 \pi / 40 \mathrm{rad}$. It is not possible to consider the tower to be axisymmetric unless the legs are approximated as a uniform ring. Nevertheless the axisymmetric assumption has been made in almost all analyses of cooling towers performed to date, ${ }^{1,2}$ as it is impracticable to perform calculations in sufficient detail to include all the legs simultaneously. However, an analysis of the sector containing 1 pair of legs shown in Figure 1 is perfectly feasible. The methods described in this paper show how a series of analyses of this one sector could be used to obtain an analysis of the complete tower, without introducing any additional approximations.

In this paper the free vibration of rotationally periodic structures is considered initially. These are frequently analysed using the finite element method, and this paper shows how such an analysis can be very considerably simplified for such structures (alternatively, how considerably more complicated structures than hitherto can be treated). However, the method proposed in this paper is not confined to use with finite elements, but can be used in any matrix 
analysis of the structure, provided it is linearly elastic. Furthermore it is not confined to vibration, but can be used in other structural eigenvalue problems, such as buckling.

The finite element analysis of infinite linear periodic structures has been described by Orris and Petyt. ${ }^{3}$ A very brief description of the work on finite rotationally periodic structures described in this paper, in which the theory is considered as an extension of Orris and Petyt's work, has already been given. ${ }^{4}$ The normal modes of the structure were considered to be standing waves, for which only certain values of the complex propagation constant are allowed. In this paper an alternative description of the modes is given, and the analysis is developed to cover a wider range of problems.

In a finite element analysis of a structure, real stiffness and mass matrices, $[K]$ and $[M]$ are set up, and the eigenvalue equation

$$
\left([K]-\omega^{2}[M]\right)\{u\}
$$

is then solved to give the natural frequencies $\omega$ and eigenvectors $\{u\}$ of the system. There are many computer programs available for carrying out this process. However, for complicated rotationally periodic structures it may be impracticable to solve equation (1) directly because of limitations on the number of degrees-of-freedom which can be handled by eigenvalue subroutines.

In section 'Rotating mode shapes' of this paper it is shown how the eigenvectors of equation (1) can be expressed as rotating eigenvectors, for rotationally periodic structures. Section 'Use of complex constraints' shows how the phase relationship between adjacent substructures undergoing a rotating vibration, can be exploited, by the use of complex constraints, so that a modified form of equation (1) is solved for a single substructure. No additional approximations are introduced using this technique. Some examples of the use of the method are given in section 'Examples'.

After the discussion of the free vibration problem, this paper considers the direct solution of the forced damped vibration problem. Static problems are of course a special case of this. For steady state conditions, the equation of motion becomes:

$$
\{F\} \mathrm{e}^{\mathrm{i} \omega t}=\left([K]+\mathrm{i}[C]-\omega^{2}[M]\right)\{u\} \mathrm{e}^{\mathrm{i} \omega t}
$$

where $[K],[C]$ and $[M]$ are the real stiffiness, damping and mass matrices, and $\{F\}$ and $\{u\}$ are complex force and displacement vectors. It is assumed that the excitation is at an angur frequency $\omega$. There are many programs available for assembling the stiffness, mass and damping matrices of complete structures, and solving equation (2) for $\{u\}$.

The forced vibration method is based on a consideration of the response of a structure to a rotating force distribution.

It is possible to decompose any arbitrary force, rotating or not, into rotating components.

In this paper we first show how any rotating force acting on the complete structure can be described in terms of a series of components acting on only one substructure. This is then generalized to include stationary forces or any arbitrary force distribution. We then show how it is only necessary to solve a modified form of equation (2) for one substructure to obtain the response to each component of force. Just as in the free vibration case, it is thus possible to deal with considerably more complex structures than would be the case if the whole structure had to be considered at one time.

MacNeal $e t \mathrm{al}^{5}$ have described a facility similar to that discussed in this paper, which they have incorporated into the program NASTRAN. Their description of the method uses real arithmetic throughout, whereas in the present paper complex arithmetic is used. This enables the components into which a general applied force is split to be interpreted physically as 
members of a series of rotating forces, which the present author feels increases the understanding of the nature of these components. Such an interpretation emphasises that, for some structures, many of the force components are completely missing, which considerably reduces the computational effort required. The equations describing the method are considerably simplified when complex arithmetic is used, as it is not necessary to consider sine and cosine components of force separately.

MacNeal et $a l^{5}$ consider rotationally periodic structures for which each substructure is connected only to the substructures on its immediate right and left. However in this paper, the treatment is generalized so that each substructure can be mechanically connected to any number of the other substructures, so long as the connections are rotationally periodic.

\section{ROTATING MODE SHAPES}

\section{Classification of mode shapes}

Orris and Petyt's paper on infinite linear periodic structures lists references to other work in that field. The vibrations of such structures are normally described in terms of the propagation of free waves. These vibrations can occur at any frequency in a number of allowable bands. The free vibrations of rotationally periodic structures, however, occur only at certain discrete frequencies. It is possible to obtain these by consideration of the standing waves in the structure using a modification of the infinite linear periodic theory. ${ }^{4}$ However, in this paper, the conventional normal modes of a finite structure are used to describe its motion.

A rotationally periodic structure consists of $N$ identical substructures. Equation (1) is the eigenvalue equation for the complete structure. The total number of degrees-of-freedom in the structure is $N J$. The degrees-of-freedom are ordered so that the $J$ degrees-of-freedom of the first substructure are followed by $J$ degrees-of-freedom on the second substructure, and so on. An eigenvector of the whole structure, $\{u\}$, can be written

$$
\{u\}=\left\{u^{(1)} \quad u^{(2)} \quad u^{(3)} \ldots u^{(N)}\right\}^{\mathrm{T}}
$$

where $\left\{u^{(i)}\right\}$ is a vector of length $J$ containing the displacements associated with the $J$ degrees-of-freedom of the $j$ th substructure. The $\left\{u^{(i)}\right\}$ are all real.

With axisymmetric structures, it is found that most modes of vibration occur in degenerate orthogonal pairs. This is because, if a mode has a maximum deflection at some point on the structure, it is clearly possible, because of the axisymmetric nature of the structure, to rotate the mode shape through any angle and not change the frequency of vibration. A similar effect is found with rotationally periodic structures. The possible mode shapes, $\{u\}$, fall into three classes, depending on the relationship between the shapes for individual substructures. These are:

(a) Each substructure has the same mode, shape as its neighbours, i.e.

$$
u^{(j)}=u^{(j+1)} \quad(\text { all } j)
$$

(b) Each substructure has the same mode shape as its neighbours, but is vibrating in antiphase with them, i.e.

$$
u^{(i)}=-u^{(i+1)} \quad(\text { all } j)
$$

(c) All other possible mode shapes.

Modes of class (a) or (b) do not exibit degeneracy (except for any 'accidental' degeneracy which may occur if an unrelated mode shape is associated with the same natural frequency). 
For class (a) modes, the eigenvector $\{u\}$ can be written

$$
\{u\}=\left\{u^{(1)} \quad u^{(1)} \quad u^{(1)} \ldots u^{(1)}\right\}^{\mathrm{T}}
$$

and it is obvious that rotating the mode shape through any integral number of substructures leaves it unchanged, so that no other mode shape is needed to describe the rotation. Class (b) modes can exist only if $N$ is even. $\{u\}$ has the form:

$$
\{u\}=\left\{u^{(1)}-u^{(1)} u^{(1)} \ldots u^{(1)}-u^{(1)}\right\}^{\mathrm{T}}
$$

Clearly rotation of the mode shape through an even number of substructures leaves it unchanged. When it is rotated through an odd number of substructures, it becomes $-\{u\}$. This does not, however, represent a new mode shape, but a change of phase of $\pi$ in the vibrations. All other mode shapes fall into class (c), and exhibit two-fold degeneracy, and must be considered in greater detail.

\section{Orthogonal pairs of eigenvectors}

A class (c) eigenvector $\{u\}$ has $u^{(j)} \neq u^{(i+1)}$ and $u^{(i)} \neq-u^{(i+1)}$. It will be assumed that it is normalized so that $\{u\}^{\mathrm{T}}\{u\}=1$. Since all the substructures are identical, the deflected shape $\left\{u^{\prime}\right\}$ obtained by rotating $\{u\}$ round one substructure, is also an eigenvector, distinguishable from $\{u\}$, and with the same eigenvalue. It is not, however, orthogonal to $\{u\}$ in general. There must be a normalized eigenvector, $\{\bar{u}\}$, orthogonal to $\{u\}$, with the same eigenvalue, to enable any eigenvectors other than $\{u\}$ to exist with this eigenvalue. $\left\{u^{\prime}\right\}$ has the form

$$
\left\{u^{\prime}\right\}=\left\{u^{(N)} \quad u^{(1)} \quad u^{(2)} \ldots u^{(N-1)}\right\}^{\mathrm{T}}
$$

and is normalized so that $\left\{u^{\prime}\right\}^{\mathrm{T}}\left\{u^{\prime}\right\}=1$. $\left\{u^{\prime}\right\}$ can be expressed as a linear combination of the two orthogonal eigenvectors $\{u\}$ and $\{\bar{u}\}$,

$$
\left\{u^{\prime}\right\}=c\{u\}+s\{\bar{u}\}
$$

where $s$ and $c$ are constants. Now consider the eigenvector $\left\{\bar{u}^{\prime}\right\}$ orthogonal to $\left\{u^{\prime}\right\}$. This can also be expressed as a linear combination of $\{u\}$ and $\{\bar{u}\}$, as

$$
\left\{\bar{u}^{\prime}\right\}=-s\{u\}+c\{\bar{u}\}
$$

Equation (9) can be verified by expanding $\left\{u^{\prime}\right\}^{\mathrm{T}}\left\{\bar{u}^{\prime}\right\}$ :

$$
\begin{aligned}
\left\{u^{\prime}\right\}^{\mathrm{T}}\left\{\bar{u}^{\prime}\right\} & =\left(c\{u\}^{\mathrm{T}}+\mathrm{s}\{\bar{u}\}^{\mathrm{T}}\right)(-s\{u\}+c\{\bar{u}\}) \\
& =c^{2}\{u\}^{\mathrm{T}}\{\bar{u}\}-s^{2}\{\bar{u}\}^{\mathrm{T}}\{u\}-c s\{u\}^{\mathrm{T}}\{u\}+s c\{\bar{u}\}^{\mathrm{T}}\{\bar{u}\} \\
& =0
\end{aligned}
$$

where use has been made of the orthogonality and normalization of $\{u\}$ and $\{\bar{u}\}$. The vectors $\left\{u^{\prime}\right\}$ and $\left\{\bar{u}^{\prime}\right\}$ can therefore be expressed as a transformation of the vectors $\{u\}$ and $\{\bar{u}\}$, by

$$
\left\{\begin{array}{l}
u^{\prime} \\
\bar{u}^{\prime}
\end{array}\right\}=\left[\begin{array}{cc}
c \mathscr{I}_{N J} & s \mathscr{I}_{N J} \\
-s \mathscr{I}_{N J} & c \mathscr{I}_{N J}
\end{array}\right]\left\{\begin{array}{l}
u \\
\bar{u}
\end{array}\right\}=[R]\left\{\begin{array}{l}
u \\
\bar{u}
\end{array}\right\}
$$

where $\mathscr{I}_{N J}$ is the unit matrix of order $N J$. Clearly, $[R]^{j}$ is a transformation matrix that rotates both $\{u\}$ and $\{\bar{u}\}$ round $j$ substructures, and hence $[R]^{N}=\Phi_{2 N J}$, as rotation round $N$ substructures leaves the vectors unchanged. 
Since $\left\{u^{\prime}\right\}$ is normalized,

$$
\begin{aligned}
1 & =\left\{u^{\prime}\right\}^{\mathrm{T}}\left\{u^{\prime}\right\} \\
& =\left(c\{u\}^{\mathrm{T}}+s\{\bar{u}\}^{\mathrm{T}}\right)(c\{u\}+s\{\bar{u}\})
\end{aligned}
$$

or

$$
1=c^{2}+s^{2}
$$

$c$ and $s$ can therefore be written as $\cos \psi$ and $-\sin \psi$.

It is now possible to write the deflections on any one substructure in terms of the deflections on any other. For the first substructure, for example, from equations (7) and (11),

$$
\left\{\begin{array}{l}
u^{(N)} \\
\bar{u}^{(N)}
\end{array}\right\}=\left[\begin{array}{cc}
c \mathscr{I}_{J} & s \mathscr{I}_{J} \\
-s \mathscr{I}_{J} & c \mathscr{I}_{J}
\end{array}\right]\left\{\begin{array}{l}
u^{(1)} \\
\bar{u}^{(1)}
\end{array}\right\}
$$

and, for the $j$ th substructure

$$
\left\{\begin{array}{l}
u^{(j-1)} \\
\bar{u}^{(i-1)}
\end{array}\right\}\left[\begin{array}{rr}
c \mathscr{I}_{J} & s \mathscr{I}_{J} \\
-s \mathscr{I}_{J} & c \mathscr{I}_{J}
\end{array}\right]\left\{\begin{array}{l}
u^{(i)} \\
\bar{u}^{(j)}
\end{array}\right\}
$$

Repeated application of equation (14) allows the components of $\{u\}$ and $\{\bar{u}\}$ acting on any substructure to be expressed in terms of those acting on substructure 1 .

\section{Complex eigenvectors}

The vectors $\{u\}$ and $\{\tilde{u}\}$ are a pair of orthogonal normalized real eigenvectors, with the same eigenvalue, of the equation of free vibration of the structure, equation (1). Any linear combination of them also satisfies (1) for the same value of $\omega$. Conventionally, only real linear combinations are considered. However, the complex vector $\{z\}$ given by

$$
\{z\}=\{u\}+\mathrm{i}\{\bar{u}\}
$$

is a perfectly valid solution of equation (1), with the same eigenvalue as both $\{u\}$ and $\{\bar{u}\}$. Equation (11) can be rewritten in terms of $\{z\}$, as

$$
\left\{z^{\prime}\right\}=\mathrm{e}^{-\mathrm{i} \psi}\{z\}
$$

which can be verified by writing the real and imaginary parts of equation (16) separately. Since the application of the transformation of equation (16) $N$ times leaves $\{z\}$ unchanged, $\psi$ must take one of the values

$$
\psi=2 \pi n / N
$$

where $n$ is an integer. Similarly, the relationship between the components of $\{z\}$ acting on neighbouring substructures, given in terms of $\{u\}$ and $\{\bar{u}\}$ in equation (14), can be written

$$
\left\{z^{(j-1)}\right\}=\mathrm{e}^{-\mathrm{i} \psi}\left\{z^{(i)}\right\} \quad \text { or } \quad\left\{z^{(i)}\right\}=\mathrm{e}^{\mathrm{i} \psi}\left\{z^{(j-1)}\right\}
$$

Equation (18) allows the complex eigenvector acting on any of the substructures to be expressed in terms of the vector acting on only one substructure.

In the method of calculating the eigenvalues and eigenvectors described in the following section, the complex eigenvectors $\{z\}$ are extracted directly. It is helpful at this stage to assign physical meaning to them. For a real eigenvector $\{u\}$, the deflected shape of the structure at some time $t$ is usually given as $\{u\} \mathrm{e}^{\mathrm{i} \omega t}$, where it is understood that the real part of this complex 
quantity gives the instantaneous deflected shape. This is $\{u\} \cos \omega t$. Similarly $\mathscr{R}\left[\{z\} \mathrm{e}^{\mathrm{i} \omega t}\right]$ gives the instantaneous deflected shape in terms of the complex vector $\{z\}$. This can be expanded as

$$
\begin{aligned}
\mathscr{R}\left[\{z\} \mathrm{e}^{\mathrm{i} \omega t}\right] & =\mathscr{R}[(\{u\}+\mathrm{i}\{\bar{u}\})(\cos \omega t+\mathrm{i} \sin \omega t)] \\
& =\{u\} \cos \omega t-\{\tilde{u}\} \sin \omega t
\end{aligned}
$$

Now at $t=0$, equation (19) gives the deflected shape as $\{u\}$, but at $\omega t=\psi$, equation (19) gives

$$
\mathscr{R}\left[\{z\} \mathrm{e}^{\mathrm{i} \omega t}\right]=\{u\} \cos \psi-\{\bar{u}\} \sin \psi
$$

and, from equation (11), this is $\left\{u^{\prime}\right\}$. At $t=\psi / \omega$ the deflected shape of the structure is therefore the same as at $t=0$, except that it has rotated round one substructure. The complex eigenvectors $\{z\}$ therefore describe rotating modes of vibration of the structure, in which the same instantaneous deflected shape reappears after successive intervals of time $t=\psi / \omega$, rotated round an additional substructure each time.

It may be objected that the eigenvectors $\{z\}$ do not satisfy one of the classical requirements for a normal mode of vibration, that all points on the structure should be in phase or antiphase with each other. $\{z\}$ is a combination of two such modes, $\{u\}$ and $\{\bar{u}\}$, in the form $\{z\}=$ $\{u\}+\mathrm{i}\{\bar{u}\}$. There is thus a phase difference of $\pi / 2$ between $\{u\}$ and $\{\bar{u}\} .\{z\}$ contains all the information given by both $\{u\}$ and $\{\bar{u}\}$ separately. Although it is complex, it is, mathematically, a valid solution of equation (1), and the two conventional modes $\{u\}$ and $\{\bar{u}\}$ can readily be extracted from it.

If $\{z\}$ corresponds to a clockwise rotation of the vector $\{u\}$, there is another possible combination of $\{u\}$ and $\{\bar{u}\}$ to form a complex vector $\{\bar{z}\}$, which corresponds to anticlockwise rotation. $\{\bar{z}\}$ is given by

$$
\{\bar{z}\}=\{u\}-\mathrm{i}\{\bar{u}\}
$$

$\{\bar{z}\}$ is orthogonal to $\{z\}$ since $\{\bar{z}\}^{* \mathrm{~T}}\{z\}=0$, which may readily be verified. Note that $\{\bar{z}\}=\{z\}^{*}$.

\section{Values of $n$}

The value of $n$ used to define $\psi$ in equation (17) will now be discussed. $n$ depends on the particular rotating mode shape $\{z\}$ which is being considered, and is a measure of the periodicity of the mode. Clearly, from equation (17), $\psi$ may have $N$ possible independent values. If $n \neq 0$, and $N / n$ is an integer, $p$, then $\{u\},\{\bar{u}\}$ and $\{z\}$ all repeat themselves identically after $p$ substructures. For example if $N=6, n=2$, then $p=3$, and all three vectors are of the form

$$
\begin{aligned}
& u^{(4)}=u^{(1)} \\
& u^{(5)}=u^{(2)} \\
& u^{(6)}=u^{(3)}
\end{aligned}
$$

For $N=6, n=1, p=6$, and there is no such relationship between the $u^{(j)}$ 's. However, if $N / n$ is not an integer there is not a relationship as simple as equation (22). If $N=7$ and $n=2, N / n=$ 3.5. This implies that the deflected shape repeats after every 3.5 substructures, which in general is not possible. However, if the deflected shape is $\{u\}$ at $t=0$, then after a time 
$t=\psi / 2 \omega$ (that is, half the time it takes for the deflected shape to move round one substructure) $\{u\}$ will appear to have moved round four substructures. $\{u\}$ may thus be thought of as having a latent periodicity of 3.5 substructures, which manifests itself when it is rotated through half a substructure.

The discussion of complex eigenvectors $\{z\}$ has been concerned with class $(c)$ modes of the structure. However, class (a) and (b) modes may be expressed in a similar way. As there is no orthogonal mode in either of these cases, $\{\bar{u}\}=0 .\{z\}$ is therefore a complex vector, all the elements of which lie on the real axis. For class (a), $\psi=0$, and for class (b), $\psi=\pi$. The corresponding values of $n$ are 0 and $N / 2$. Clearly $N / 2$ is only an integer if $N$ is even, so class (b) modes cannot exist with $N$ odd.

As the number of independent values of $n$ is $N$, the possible independent values of $\psi$, for $N$ even, may be written

$$
-2 \pi(N / 2-1) / N, \ldots-4 \pi / N,-2 \pi / N, 0,2 \pi / N, 4 \pi / N, \ldots 2 \pi(N / 2-1) / N, \pi
$$

and for $N$ odd

$$
-2 \pi(N-1) / 2 N, \ldots-2 \pi / N, 0,2 \pi / N, 4 \pi / N, \ldots 2 \pi(N-1) / 2 N
$$

However, the negative values of $\psi$ correspond to anticlockwise rotation and the positive values to clockwise. Since all the anticlockwise eigenvectors are the orthogonal pairs of clockwise ones, they can be generated, once the clockwise vectors $\{z\}$ are known, simply by generating the complex conjugates of $\{z\}$. To obtain all the possible modes of vibration of the structure, therefore, it is necessary to find the modes corresponding, for $N$ even, to the $N / 2+1$ values of $\psi$ :

$$
0,2 \pi / N, 4 \pi / N, \ldots 2 \pi(N / 2-1) / N, \pi
$$

and, for $N$ odd, to the $(N+1) / 2$ values of $\psi$

$$
0,2 \pi / N, 4 \pi / N \ldots 2 \pi(N-1) / 2 N
$$

\section{Summary}

In this section we have seen how most possible modal shapes of a rotationally periodic structure exist in orthogonal pairs. By combining these into complex eigenvectors $\{z\}$, they may be considered as rotating modal shapes. The displacement of any substructure can be related to the displacement of one particular substructure by a phase angle $\psi$. The non-rotating class (a) and (b) modes can be considered as special cases of the rotating modes, with $\psi=0$ and $\pi$. There are $N$ possible values of $\psi$, but only $(N / 2)+1($ even $N)$ or $(N+1) / 2$ (odd $N)$ of these represent different mode shapes, the remainder corresponding to similar shapes rotating in the opposite sense.

Any complex mode shape of the structure can be expressed in terms of the deflected shape of just one substructure, by use of the appropriate value of $\psi$. As the number of values of $\psi$ is limited it is possible to examine each in turn. In the following section, it is shown how, for a 
given value of $\psi$, it is possible to calculate all the corresponding rotating mode shapes of one substructure, and thence of the whole structure, from the mass and stiffness matrices of one substructure without any approximation. By repeating this for every possible value of $\psi$, it is possible to obtain all the mode shapes of the complete structure.

\section{USE OF COMPLEX CONSTRAINTS}

\section{Equation of free vibration}

The rotationally periodic structure is divided into $N$ identical substructures, each of which contains $J$ degrees-of-freedom. However, each substructure is connected to a number of other substructures. This number is at least 2 and may include all the other substructures. The stiffness matrix for one substructure can be expressed as a number of $J \times J$ submatrices. For the $i$ th substructure these submatrices are $K_{i i}^{(i)}$, which represents the interaction between the $J$ degrees-of-freedom in the substructure, and up to $N-1$ triads of submatrices $K_{i j}^{(i)}, K_{j i}^{(i)}$ and $K_{j i}^{(i)}$. These triads represent the interaction between the $i$ th and $j$ th substructure. The equation of motion of the complete structure, equation (1), can therefore be expanded to give equation (23).

$$
\left\{\left[\begin{array}{ccccc}
\sum_{i=1}^{N} K_{11}^{(i)} & K_{12}^{(1)}+K_{12}^{(2)} & K_{13}^{(1)}+K_{13}^{(3)} & \ldots & K_{1 N}^{(1)}+K_{1 N}^{(N)} \\
K_{21}^{(2)}+K_{21}^{(1)} & \sum_{i=1}^{N} K_{22}^{(i)} & K_{23}^{(2)}+K_{23}^{(3)} & \ldots & K_{2 N}^{(2)}+K_{2 N}^{(N)} \\
K_{31}^{(3)}+K_{31}^{(1)} & K_{32}^{(3)}+K_{32}^{(2)} & \sum_{i=1}^{N} K_{33}^{(i)} & \ldots & K_{3 N}^{(3)}+K_{3 N}^{(N)} \\
\vdots & \vdots & & & \vdots \\
K_{N 1}^{(N)}+K_{N 1}^{(1)} & K_{N 2}^{(N)}+K_{N 2}^{(2)} & K_{N 3}^{(N)}+K_{N 3}^{(3)} & \ldots & \sum_{i=1}^{N} K_{N N}^{(i)}
\end{array}\right]\right.
$$$$
-\omega^{2}\left[\begin{array}{ccccc}
\sum_{i=1}^{N} M_{11}^{(i)} & M_{12}^{(1)}+M_{12}^{(2)} & M_{13}^{(1)}+M_{13}^{(3)} & \ldots & M_{1 N}^{(1)}+M_{1 N}^{(N)} \\
M_{21}^{(2)}+M_{21}^{(1)} & \sum_{i=1}^{N}+M_{22}^{(i)} & M_{23}^{(2)}+M_{23}^{(3)} & \ldots & M_{2 N}^{(2)}+M_{2 N}^{(N)} \\
M_{31}^{(3)}+M_{31}^{(1)} & M_{32}^{(3)}+M_{32}^{(2)} & \sum_{i=1}^{N} M_{33}^{(i)} & \ldots & M_{3 N}^{(3)}+M_{3 N}^{(N)} \\
\vdots & \vdots & & \vdots \\
M_{N 1}^{(N)}+M_{N 1}^{(1)} & M_{N 2}^{(N)}+M_{N 2}^{(2)} & M_{N 3}^{(N)}+M_{N 2}^{(3)} & \ldots & \sum_{i=1}^{N} M_{N N}^{(i)}
\end{array}\right]\left\{\begin{array}{c}
z^{(1)} \\
z^{(2)} \\
z^{(3)} \\
\vdots \\
\left.z^{(N)}\right)
\end{array}\right\}=
$$ 


\section{Solution with complex constraints}

The first row of equation (23) can be written

$$
\begin{aligned}
& \left\{\left[\begin{array}{ll}
\sum_{i=1}^{N} K_{11}^{(i)} & K_{12}^{(1)}+K_{12}^{(2)} \ldots K_{1 N}^{(1)}+K_{1 N}^{(N)}
\end{array}\right]\right. \\
& \left.\quad-\omega^{2}\left[\begin{array}{ll}
\sum_{i=1}^{N} M_{11}^{(i)} & M_{12}^{(1)}+M_{12}^{(2)} \ldots M_{1 N}^{(1)}+M_{1 N}^{(N)}
\end{array}\right]\right\}\left\{\begin{array}{c}
z^{(1)} \\
z^{(2)} \\
\vdots \\
z^{(N)}
\end{array}\right\}=0
\end{aligned}
$$

However, since all the substructures are identical

$$
K_{11}^{(j)}=K_{(N+2-j)(N+2-i)}^{(1)} \text { for } j=2,3, \ldots, N
$$

and hence

$$
\sum_{i=1}^{N} K_{11}^{(i)}=\sum_{i=1}^{N} K_{i i}^{(1)}
$$

with a similar relationship applying for the mass matrices.

The corresponding connexion terms between substructures are also identical, so

$$
K_{1 j}^{(j)}=K_{(N+2-i) 1}^{(1)} \text { for } j=2,3, \ldots, N
$$

$z^{(2)}, z^{(3)}$ up to $z^{(N)}$ can be eliminated from equation (24) by using equation (18), and substituting from equations (25) and (26) into equation (24) gives

$$
\begin{aligned}
& \left\{\left[\sum_{j=1}^{N} K_{j i}^{(1)}+\sum_{j=2}^{N} K_{1 j}^{(1)} \mathrm{e}^{\mathrm{i}(i-1) \psi}+\sum_{i=2}^{N} K_{(N+2-j)}^{(1)} \mathrm{e}^{\mathrm{i}(j-1) \psi}\right]\right. \\
& \left.-\omega^{2}\left[\sum_{j=1}^{N} M_{i j}^{(1)}+\sum_{j=2}^{N} M_{1 j}^{(1)} \mathrm{e}^{\mathrm{i}(j-1) \psi}+\sum_{j=2}^{N} M_{(N+2-j) 1}^{(1)} \mathrm{e}^{\mathrm{i}(i-1) \psi}\right]\right\}\left\{z^{(1)}\right\}=0
\end{aligned}
$$

where $\psi$ has one of the possible values discussed in section 'Rotating mode shapes'. If we now decide to search specifically for those modes corresponding to a particular allowable value of $\psi, \psi$ can be fixed at this point.

Now by putting $k=N+2-j$ and observing that

$$
\mathrm{e}^{\mathrm{i}(j-1) \psi}=\mathrm{e}^{\mathrm{i}(N+1-k) \psi}=\mathrm{e}^{\mathrm{i} N \psi} \mathrm{e}^{\mathrm{i}(1-k) \psi}=\mathrm{e}^{-\mathrm{i}(k-1) \psi}
$$

by virtue of the fact that $N \psi$ is a multiple of $2 \pi$, we can write

$$
\sum_{i=2}^{N} K_{(N+2-j) 1}^{(1)} \mathrm{e}^{\mathrm{i}(j-1) \psi}=\sum_{k=2}^{N} K_{k 1}^{(1)} \mathrm{e}^{-\mathrm{i}(k-1) \psi}
$$

and replacing the dummy suffix $k$ by $j$ equation (27) then gives

$$
\begin{aligned}
& \left\{\left[\sum_{i=1}^{N} \mathrm{~K}_{\mathrm{ji}}^{(1)}+\sum_{j=2}^{N} \mathrm{~K}_{1 \mathrm{j}}^{(1)} \mathrm{e}^{\mathrm{i}(j-1) \psi}+\sum_{j=2}^{N} K_{j 1}^{(1)} \mathrm{e}^{-\mathrm{i}(j-1) \psi}\right]\right. \\
& -\omega^{2}\left[\sum_{j=1}^{N} M_{j j}^{(1)}+\sum_{i=2}^{N} M_{1 i}^{(1)} \mathrm{e}^{\mathrm{i}(j-1) \psi}+\sum_{j=2}^{N} M_{j 1}^{(1)} \mathrm{e}^{-i(j-1) \psi}\right]\left\{z^{(1)}\right\}=0
\end{aligned}
$$


Equation (28) is an eigenvalue equation containing only the $J$ degrees-of-freedom in substructure 1, and it is not coupled to any degrees-of-freedom in other substructures. It can therefore be solved to give $J$ eigenvalues $\omega^{2}$ and associated eigenvectors $\left\{z^{(1)}\right\}$, and equation (18) can then be used to generate the $J$ eigenvectors $\{z\}$ which act on the complete structure. The $\{z\}$ eigenvectors are complex, and the associated orthogonal pairs of real eigenvectors, $\{u\}$ and $\{\bar{u}\}$, can be obtained from the real and imaginary parts of $\{z\}$.

The conventional eigenvalue problem encountered in structural mechanics involves two real matrices, as in equation (1). The eigenvalue problem in equation (28), however, involves complex mass and stiffness matrices. However, it may be seen by inspection of equation (28) that both matrices are Hermitian, that is, that the real parts are symmetric and the imaginary parts are skew-symmetric. It is a property of Hermitian matrices that the eigenvalues are real, but the eigenvectors are complex. Solution of equation (28) therefore leads to real values of $\omega^{2}$, but the eigenvectors $\{z\}$ are complex, as expected for rotating mode shapes.

Solution of equation (28) gives $J$ eigenvalues. There are $N$ possible values of $\psi$, so there are $N J$ eigenvalues obtainable if equation (27) is set up and solved for every value of $\psi$. This is the same as the number of degrees-of-freedom of the entire structure, and hence the same as the number of eigenvalues of equation (1). All of the eigenvectors of the complete structure can therefore be obtained from $N$ analyses of one substructure. As mentioned in subsection 'Values of $n$ ', negative values of $\psi$ correspond to eigenvectors with the same frequencies and mode shapes as, but rotating in the opposite sense to, eigenvectors with positive values of $\psi$. It is not therefore necessary to solve equation (28) for negative values of $\psi$.

The complex mass and stiffness matrices for one substructure in equation (28) can readily be set up. The matrices are formed for a single substructure, including the interconnexion terms to all the other substructures. The stiffness matrix $\left[K_{0}\right]$, has the form

$$
\left[K_{0}\right]=\left[\begin{array}{ccccc}
K_{11}^{(1)} & K_{12}^{(1)} & K_{13}^{(1)} & \ldots & K_{I N}^{(1)} \\
K_{21}^{(1)} & K_{22}^{(1)} & 0 & \ldots & 0 \\
K_{31}^{(1)} & 0 & K_{33}^{(1)} & \ldots & 0 \\
\vdots & \vdots & \vdots & & \vdots \\
K_{N 1}^{(1)} & 0 & 0 & \ldots & K_{N N}^{(1)}
\end{array}\right]
$$

This has $N J$ degrees-of-freedom if none of the rows contain all zero terms. It is reduced to a $J$ degree-of-freedom system by applying complex constraints of the form of

$$
\left\{z^{(i)}\right\}=\mathrm{e}^{\mathrm{i}(j-1) \psi}\left\{z^{(1)}\right\}
$$

The constrained stiffness matrix $\left[K_{p}\right]$ is obtained by applying a transformation to $\left[K_{0}\right]$ :

$$
\left[K_{p}\right]=\left[T^{\mathrm{T}^{*}}\right]\left[K_{0}\right][T]
$$

where

$$
[T]=\left[\begin{array}{c}
\mathscr{I}_{J} \\
\mathrm{e}^{\mathrm{i} \psi \mathscr{I}_{J}} \\
\mathrm{e}^{2 \mathrm{i} \Psi} \mathscr{\mathscr { I }}_{J} \\
\vdots \\
\mathrm{e}^{\mathrm{i}(N-1) \psi_{\mathscr{T}}}
\end{array}\right]
$$


and $\left[T^{\mathrm{T}^{*}}\right]$ is its conjugate transpose. It may readily be verified that $\left[K_{p}\right]$ evaluated from equations (29) and (31) is the stiffness matrix used in equation (28). The mass matrix may be obtained in the same manner.

For most practical problems, each substructure will only be connected to a small number of other substructures. Many of the submatrices in equation (29) may therefore have all zero terms. If only a few degrees-of-freedom are joined on those substructures which are connected, many of the rows of the submatrices which remain in equation (29) will also be zero. The size of $\left[K_{0}\right]$ may not therefore be particularly large, and the computational effort required to evaluate $\left[K_{p}\right]$ from equation (31) can thus be considerably reduced.

\section{Computer time and storage}

It is interesting to compare the computer time and storage required in a solution of a structural eigenvalue problem directly, solving equation (1), and using complex constraints on a single substructure, solving equation (27). The major part of the solution is the calculation of the eigenvalues once $[K]$ and $[M]$ have been set up. Many eigenvalue subroutines take a central processing time (CPU time) proportional to the numbers of degrees-of-freedom cubed. To solve equation (1) the time is therefore proportional to $N^{3} J^{3}$. To use complex constraints, only $J$ degrees-of-freedom are used, but each multiplication is complex, and thus equivalent to about four real multiplications. The time to extract the eigenvalues for one value of $\psi$ is thus $\sim 4 J^{3}$ For an even value of $N$ (which is the worse case) there are $N / 2+1$ values of $\psi$ (ignoring negative values). The CPU time to extract all the eigenvalues of the structure is therefore proportional to $(2 N+4) J^{3}$. The ratio of CPU times for analysing the whole structure, and for an analysis using complex constraints, is $N^{3} /(2 N+4)$. Even for quite small values of $N$ this ratio is substantial, e.g. for $N=6$ it is $13 \cdot 5$.

To store the matrices for the whole structure, core storage proportional to $N^{2} J^{2}$ is required. When complex constraints are used, the number of array elements to be stored is $J^{2}$, but as these are complex the storage required is proportional to $2 J^{2}$. The ratio of core storage for the mass and stiffness matrices required for the whole structure, and for one substructure using complex constraints, is thus $N^{2} / 2$. Again, this ratio is large even for small values of $N$, e.g. for $N=6$ it is 18 .

With many in-core eigenvalue subroutines core storage limitations put an upper limit of about 200 degrees-of-freedom on the size of matrices that can be solved directly (using equation (1)). This means that a rotationally periodic structure with more than a very few substructures cannot be analysed at all, except by gross over-simplification, if the complete structure is included. With the use of complex constraints the storage limit is about $200 / \sqrt{2}$, or 140, degrees-of-freedom, after application of the constraints. As only one substructure is represented, however, a fairly detailed substructure can be considered.

\section{EXAMPLES}

To illustrate the use of the method in a practical problem, its application to the calculation of the normal modes of a fully supported alternator end-winding is described in this section. The end-winding consists of 48 identical pairs of conductors. Each conductor leaves the cylindrical core of the machine (see the schematic idealization in Figure 2), describes an involute of a circle on a conical surface to the nose region, at which point it is connected to another conductor, lying on a different conical surface, which returns to the core. Each conductor has interconnexions along the whole of its length with its nearest neighbours, and also with the 


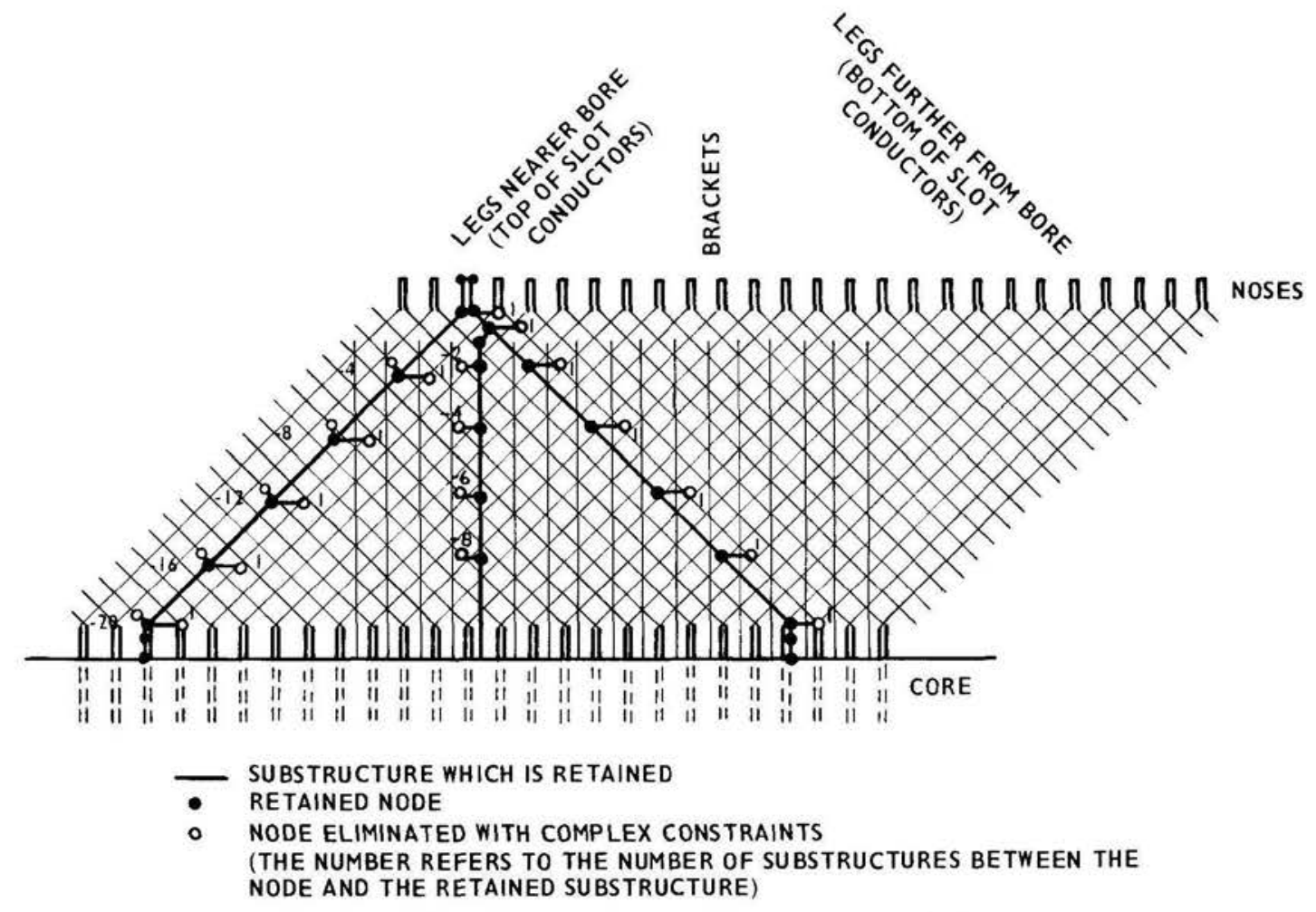

Figure 2. Schematic idealization of alternator end winding

conductors which cross it on the other conical surface. In addition, for every two conductors there is a support bracket spanning several conductors and fixed to the outer layer.

It has been found that one conductor can be adequately represented by about 18 beam finite elements. The nodes are shown schematically in Figure 2. There are 6 degrees-of-freedom for each node, giving 102 unconstrained degrees-of-freedom per conductor. In order to simplify the structure, it is assumed that each bracket can be replaced by two brackets, each of half the correct stiffness, so that the number of brackets and the number of conductors are equal. The end-winding then consists of 48 identical substructures, each including one conductor, a support bracket, and one set each of inter-coil and inter-layer connexions. One such substructure is shown in Figure 2.

The nodes of the first boundary of the substructure, and internal nodes, are shown as solid circles. The second boundary nodes, which form part of other substructures, are shown as open circles. By each of these there is an integer $n$. This represents the number of substructures through which it is necessary to rotate the original substructure in order that the open circle node lies on the original substructure.

If calculations of the normal modes of the complete structure are performed for a particular value of $\psi$, as defined in previous sections, it is necessary to consider only the substructure shown in Figure 2. Each open circle node has a constraint applied, of the form

$$
u^{\prime \prime}=u^{\prime} \mathrm{e}^{\mathrm{i} n \psi}
$$


relating it to the corresponding solid circle node. It is therefore possible to perform calculations for the complete machine, using 120 complex degrees-of-freedom. To analyse the complete machine, without using complex constraints, would take $120 \times 48$, or 5760 , real degrees-of-freedom, which is impracticable for a free vibration problem.

Table I shows some of the natural frequencies of an end winding which has been analysed using complex constraints, for various values of $n$. For comparison, the frequencies of a coil, without any supports of connexions to other coils, are shown. It can be seen that the influence of the supports on the behaviour of this machine is considerable.

Table I. Natural frequencies (in $\mathrm{Hz}$ ) of a supported end winding

\begin{tabular}{rrrc}
\hline$n=1$ & $n=2$ & $n=3$ & Unsupported \\
\hline $24 \cdot 6$ & $36 \cdot 7$ & $47 \cdot 7$ & $5 \cdot 94$ \\
$34 \cdot 5$ & $46 \cdot 7$ & $56 \cdot 0$ & $15 \cdot 1$ \\
$51 \cdot 1$ & $55 \cdot 8$ & $64 \cdot 5$ & $25 \cdot 8$ \\
$68 \cdot 6$ & $72 \cdot 3$ & $78 \cdot 3$ & $32 \cdot 9$ \\
$83 \cdot 3$ & $83 \cdot 8$ & 86.6 & $45 \cdot 3$ \\
$104 \cdot 9$ & $109 \cdot 0$ & $115 \cdot 6$ & 56.7 \\
$112 \cdot 8$ & $117 \cdot 2$ & $123 \cdot 7$ & $67 \cdot 3$ \\
\hline
\end{tabular}

Another example of a structure which has been analysed using the methods described in this paper is the cooling tower shown in Figure 1. The substructure was idealized with five of DebNath's shell elements ${ }^{7}$ together with two beam elements representing the pair of legs. All the degrees-of-freedom on one boundary were related to those on the other using complex constraints. Full details of the calculations, ${ }^{8}$ and of measurements on both a model and a full scale structure, ${ }^{9}$ are given elsewhere, but the results are summarized in Table II. It can be seen that the agreement between model and calculated frequencies is excellent. The lower modes of the full scale structure have slightly lower frequencies than predicted. This is because of the effects of foundation flexibility. ${ }^{10}$

After application of the constraints, the substructure contains 72 degrees-of-freedom, so that the extraction of the eigenvalues is a simple and inexpensive procedure. However, to analyse the full structure, using an equivalent idealization, would require $40 \times 72$, or 2880 degrees-offreedom, which would be a very complex and expensive problem.

Table II. Comparison of calculated and measured cooling tower natural frequencies (in $\mathrm{Hz}$ ). The model results have been converted to equivalent full-scale values

\begin{tabular}{cccccc}
\hline & & \multicolumn{5}{c}{ Measured } \\
\cline { 3 - 6 } Mode & Calculated & Model & \% discrepancy & Full scale & $\%$ discrepancy \\
\hline & 1.155 & 1.159 & -0.3 & 1.081 & 6.8 \\
2 & 1.241 & 1.272 & -2.4 & 1.175 & 5.6 \\
3 & 1.399 & 1.369 & 2.2 & 1.301 & $7 \cdot 5$ \\
4 & 1.394 & 1.452 & -4.0 & 1.410 & -1.1 \\
5 & 1.630 & 1.620 & 2.0 & 1.622 & 0.5 \\
6 & 1.696 & 1.676 & 1.2 & 1.679 & 1.0 \\
7 & 1.924 & 1.950 & -1.3 & 1.978 & -2.7 \\
\hline
\end{tabular}


Another structure which has been analysed using this method is a 151-bladed turbine wheel. The mesh of 20-noded isoparametric elements used for one substructure, which represents a single blade rigidly clamped at the disk and coupled to its neighbours at the tip, is shown in Figure 3. The mesh contains 77 elements and 605 nodes. To reduce the number of degrees-offreedom to an acceptable level, the nodal condenstation technique ${ }^{6}$ was used. It is essential that all degrees-of-freedom common to more than one substructure are retained as masters, and that a sufficient number are retained on the remainder of the structure to ensure sufficient accuracy. 123 master degrees-of-freedom were retained in the analysis. The node condensation was performed first, followed by the application of complex constraints for each value of $n$, and extraction of the eigenvalues. This means that the expensive node condensation stage need only be performed once.
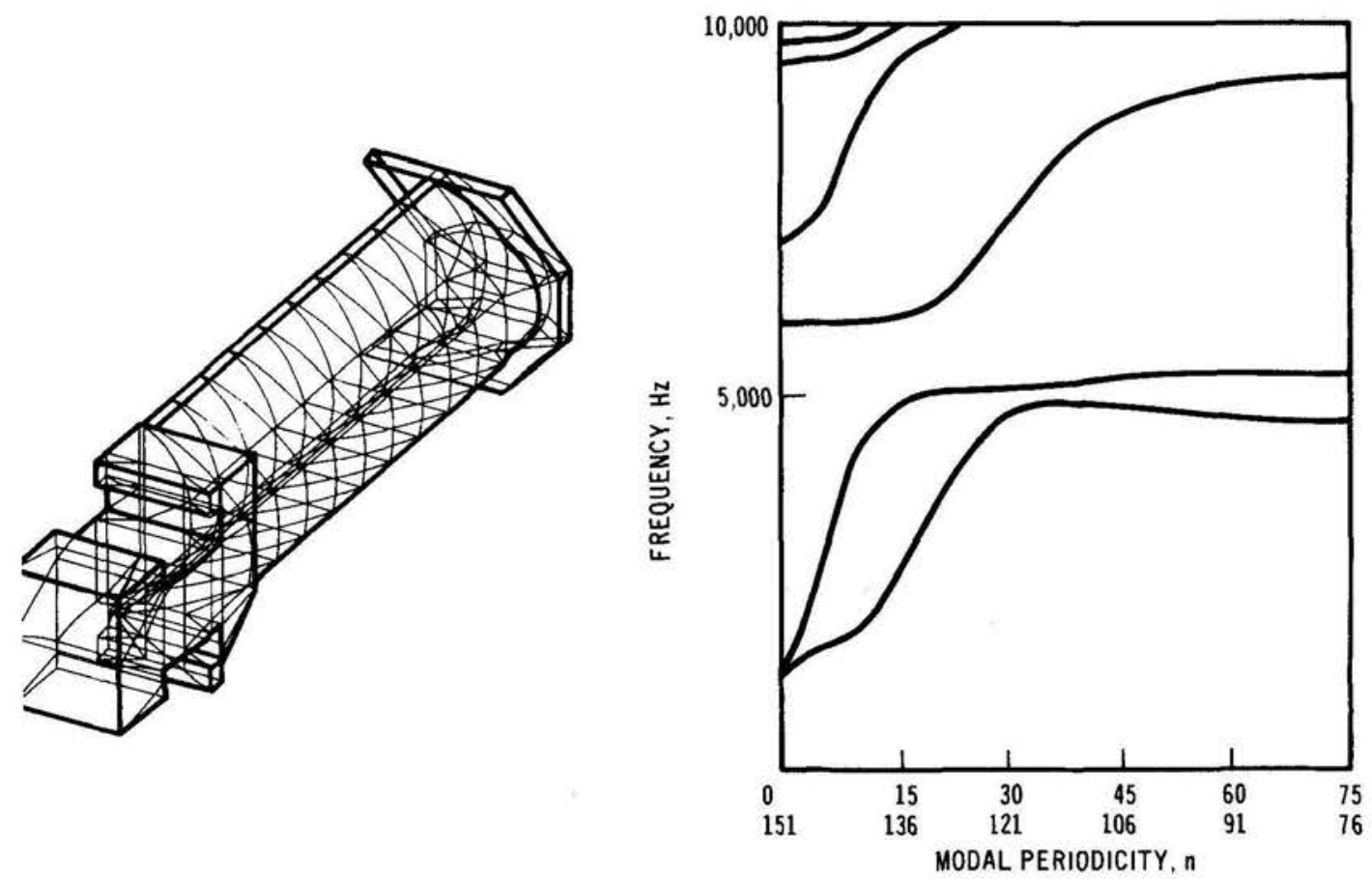

Figure 3. The mesh shows one blade, with its root and shroud, of a 151-bladed turbine disk. The graph shows the calculated frequencies as a function of modal periodicity, $n$

The results of the analysis are shown in Figure 3, in the form of the frequencies plotted as a function of modal periodicity $n$. It can be seen that the existence of coupling between the blades has a major influence on the frequencies, demonstrated by their variability with $n$.

\section{FORCED VIBRATION}

\section{Rotating forces}

The previous section have shown how the free vibration problem can be solved for rotationally periodic structures using the methods of complex constraints. Forced vibration problems 
can either be solved using modal synthesis, in which case the methods described above are of use, or by direct solution. In this section we show how the direct solution can be simplified to a series of solutions on a single substructure, by decomposing the force into a series of rotating components. We first consider the decomposition of a general rotating force, and then generalize the treatment to include any arbitrary force distribution.

We first consider a rotating force acting on the complete structure. This consists of $\cdot N$ identical substructures. These are divided into $M$ groups, containing equal numbers of substructures. The force system acting on the complete structure may be called a rotating force system if the force acting on any degree-of-freedom on one substructure has the same amplitude as the force acting on the same degree-of-freedom in the $N / M$ th following substructure and there is a fixed difference between them. Thus, if $F_{k, j} \mathrm{e}^{\mathrm{i} \omega t}$ is the force acting on any degree-of-freedom $k$, in the jth substructure:

$$
F_{k, j+N / M} \mathrm{e}^{\mathrm{i} \omega t}=F_{k, j} \mathrm{e}^{\mathrm{i}(\omega t-\phi+(p-1) 2 \pi)}
$$

where $p$ is an integer. After a time $t=\phi / \omega$, the instantaneous force distribution is identical to that at $t=0$, except that it has moved round $N / M$ substructures. If the complete structure can be divided into $L$ groups, each subject to a force distribution identical in both amplitude and phase, then:

$$
F_{k, i+N / L} \mathrm{e}^{i \omega t}=F_{k, i} \mathrm{e}^{\mathrm{i}(\omega t-(\phi-(p-1) 2 \pi) M / L)}=F_{k, j} \mathrm{e}^{\mathrm{i} \omega t}
$$

so that:

$$
\phi=2 \pi(L / M+p-1)
$$

where $p=1,2, \ldots$.

We have described above how a rotating system of forces acting on a complete structure can be completely described in terms of the forces acting on a group of $N / M$ substructures. The forces on the rest of the structure are found from equation (32) where $\phi$ is given by equation (34), and $p$ may be set to 1 . However, it is convenient to express the forces acting on the group of substructures in terms of a number of components acting on a single substructure. Each of these components is a rotating force, with the same amplitude on all substructures, and with a phase difference of $\phi M / N$ between neighbouring substructures. There are $N / M$ components, associated with $N / M$ values of $\phi$, which are given by equation (34) with $p=1,2, \ldots, N / M$. If the $p$ th component, acting on the $k$ th node of the first substructure, is $A_{k, p} \mathrm{e}^{\mathrm{i} \omega t}$, then:

$$
F_{k, 1} \mathrm{e}^{\mathrm{i} \omega t}=\sum_{p=1}^{N / M} A_{k, p} \mathrm{e}^{\mathrm{i} \omega t}
$$

The phase difference between substructures is $\phi M / N$, so the $p$ th component acting on the $j$ th substructure, $A_{k, p}^{(j)}$, is, using equation (34),

$$
A_{k, p}^{(j)} \mathrm{e}^{\mathrm{i} \omega t}=A_{k, p} \exp [\mathrm{i}(\omega t-2 \pi(j-1)(L+M(p-1)) / N)]
$$

so the total force, acting on the $k$ th degree-of-freedom of the $j$ th substructure, is:

$$
F_{k, j} \mathrm{e}^{\mathrm{i} \omega t}=\sum_{p=1}^{N / M} A_{k, p} \exp [-2 \pi \mathrm{i}(j-1)(L+M(p-1)) / N] \mathrm{e}^{\mathrm{i} \omega t}
$$

Here $j$ can take the values $1,2, \ldots, N / M$.

If we define a $J \times N / M$ matrix $[F]$, where $J$ is the number of degrees-of-freedom on one substructure, as the matrix whose elements are the $F_{k, j}$ 's, and a $J \times N / M$ matrix $[A]$ of force 
components, for which the $p$ th element in the $k$ th row is $A_{k, p}$, then equation (37) can be written:

$$
[F]=\sqrt{ }(N / M)[A][\phi]
$$

where $[\phi]$ is an $N / M$ by $N / M$ matrix, for which the $j$ th entry in the $p$ th row is $\phi_{p, j}$ given by:

$$
\phi_{p, j}=\sqrt{ }(M / N) \exp [-2 \pi \mathrm{i}(j-1)(L+M(p-1)) / N]
$$

Equation (38) is a means of obtaining $[F]$, assuming $[A]$ is known; that is, if the components into which $[F]$ is divided are known. However, normally one knows the force distribution on a group of substructures, $[F]$ and does not know $[A]$. This can be obtained from:

$$
[A]=[F][\phi]^{-1}(N / M)^{-1 / 2}
$$

The matrix $[\phi]$ is orthogonal, (see Appendix I) so its inverse $[\theta]$ is given by:

$$
[\theta]=[\phi]^{-1}=[\phi]^{* \mathrm{~T}}
$$

where $[\phi]^{* \mathrm{~T}}$ is the conjugate transpose of $[\phi]$, and from equation (39), the elements of $[\theta], \theta_{p, i}$, are:

$$
\theta_{p, j}=\sqrt{ }(M / N) \exp [2 \pi i(p-1)(L+M(j-1)) / N]
$$

Equation (40) shows how a system of rotating forces acting on a rotationally periodic structure can be expressed in terms of a number of components, $A_{k, p}$, each of which describes a rotating force, which has the same amplitude on all substructures, and a constant phase relationship between adjacent substructures (see equation (36)). It is shown below how this property can be used to enable the deflections on the whole structure to be obtained from computations on a single substructure.

The decomposition of the forces described above assumed that the initial force distribution $F_{k, j} \mathrm{e}^{\mathrm{i} \omega t}$ is rotating (i.e., amplitude repeats after every $N / M$ substructures) and periodic (amplitude and phase repeat after every $N / L$ substructures). However, a non-periodic rotating force is obtained with $L=1$, and a completely general force can therefore still be expressed in terms of $N$ rotating components by setting $M=L=1$, so that the analysis puts no restrictions whatever on the spatial distribution of the force. If a number of different frequencies is present, the analysis above can be applied to each frequency component separately.

\section{Response calculations}

The response of the structure to one of the rotating force components is now considered. The vector $\left\{A_{p}\right\}$, which is the $p$ th component of the force on the first substructure, is the $p$ th column of $[A]$, found from equation (40). From equation (36) the force on the $j$ th substructure is:

$$
\left\{A_{p}^{(j)}\right\} \mathrm{e}^{\mathrm{i} \omega t}=\left\{A_{p}\right\} \exp [-2 \pi \mathrm{i}(j-1)(L+M(p-1)) / N] \mathrm{e}^{\mathrm{i} \omega t}
$$

Since the force on the $j$ th substructure (for any $j$ ) has the same amplitude, but a phase lag of $\phi=2 \pi(j-1)(L+M(p-1)) / N$ behind that on the first substructure, it follows that after a time $t=\phi / \omega$ the instantaneous force distribution over the whole structure is the same as at $t=0$, except that it has moved round $(j-1)$ substructures. Furthermore, at $t=\phi / \omega+\tau$, where $\tau$ has any value, it is the same as it is at $t=\tau$, except for being displaced by $(j-1)$ substructures. The response of the entire structure at $t=\phi / \omega+\tau$ is therefore identical to that at $t=\tau$, except for a displacement round $(j-1)$ substructures. The displacement vector for the first substructure, 
$\left\{u_{p}\right\}$, is thus related to that of the $j$ th substructure, $\left\{u_{p}^{(i)}\right\}$, by a relationship similar to equation $(43)$ :

$$
\left\{u_{p}^{(j)}\right\} \mathrm{e}^{\mathrm{i} \omega t}=\left\{u_{p}\right\} \exp [-2 \pi \mathrm{i}(j-1)(L+M(p-1)) / N] \mathrm{e}^{\mathrm{i} \omega t}
$$

Once $\left\{u_{p}\right\}$ has been found for the first substructure, equation (44) can be used to obtain the displacement on any other substructure.

Equation (44) demonstrates that the response to a rotating force vector has a similar form to the rotating mode shapes considered in the free vibration case. The same method of analysis, with the displacements on one boundary of a substructure constrained to be $\exp [-2 \pi \mathrm{i}(L+$ $M(p-1)) / N]$ times those on the other boundary, can therefore be used. The constrained dynamic stiffness matrix $\left[\mathscr{K}_{p}\right]$ for the $p$ th rotating component of the force, is found from the dynamic stiffness matrix of a single substructure, $\left[\mathscr{K}_{0}\right]$, by the application of appropriate complex constraints. The equation of motion for the $p$ th component is

$$
\left\{A_{p}\right\}=\left[\mathscr{K}_{p}\right]\left\{u_{p}\right\}
$$

which may be used to calculate the response of substructure 1 . The response at any other point may then be obtained from equation (44).

The response of the whole structure to the original force $[F]$ can be obtained by assembling a $J \times N / M$ matrix $[U]$, the $p$ th column of which contains $\left\{u_{p}\right\}$. A $J \times N / M$ matrix $[V]$ has elements $v_{k j}$, where $v_{k j}$ is the reponse of the $k$ th degree of freedom on the $j$ th substructure. [V] is obtained, from equation (38) by:

$$
[V]=\sqrt{ }(N / M)[U][\phi]
$$

The ordering of the degrees-of-freedom in the substructure analysed is immaterial. If the order differs from that assumed above, appropriate rows and columns in $\left[\mathscr{K}_{0}\right]$ and $[T]$ should be interchanged.

To illustrate the use of complex constraints on a periodic structure, the example shown in Figure 4 has been analysed. This consists of a highly idealized alternator end-winding. The structure lies in a plane, and only in-plane motions are considered. There are therefore three degrees-of-freedom at each unclamped node. There are six phase groups each containing 1 coil which runs from the core of the machine, to the nose, and back to the core. Each coil is connected to its neighbours by a ring beam at the nose. Oscillating forces of unit magnitude, and phases of $0,-120,-240$ degrees are applied at the points shown.

Initially the complete structure was analysed. A single substructure (see Figure 4) was then analysed, and the complex constraint facility described in the previous section was used on all three degrees-of-freedom on node 8 , relating them to the corresponding degrees-of-freedom of node 3 , with $\psi=-120$ degrees. For a damped forced motion, the results for substructure 1 were identical to those obtained when the whole structure was analysed. However, the analysis of the complete structure required 1.9 times as much core storage, and 4.5 times as much computer time, as the analysis using complex constraints. For more realistic, larger structures, these ratios become very much greater, rendering a complete analysis impracticable.

The method has been used to calculate the forced vibrations of the alternator end-winding shown schematically in Figure 2. It was not possible to analyse the full structure without using the complex constraint method. However, to test its correctness when applied to a large structure, a simplified structure, containing 48 coils and the connexions between adjacent coils, but no brackets or connexions between layers, was analysed using the complex constraint method, and using a full idealization. Excellent agreement between the two calculations was obtained. 


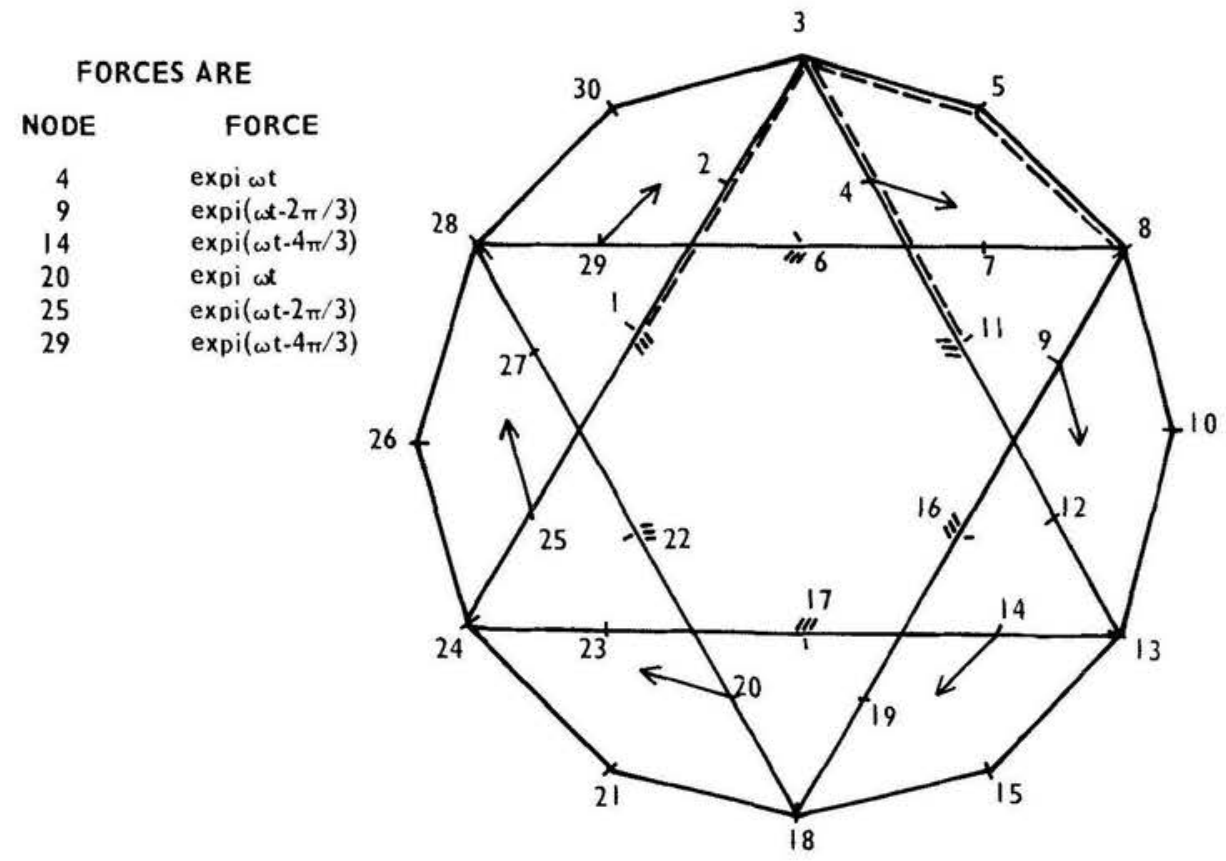

Figure 4. Simplified end winding. Nodes $1,6,11,16,17,22$ are clamped. $=--$ shows part of structure analysed with complex constraints. $\rightarrow$ shows direction of application of a force

\section{CONCLUSIONS}

A method for solving eigenvalue problems for rotationally periodic structures without introducing any additional approximations has been described. The working of the method is performed on a single substructure throughout.

The method enables an economical analysis to be performed on structures too complex to be analysed at all hitherto. The method can also be used for the direct solution of forced vibration problems, by reducing the force to a set of rotating force components.

\section{ACKNOWLEDGEMENTS}

The author thanks Mr. R. M. Woods and Mrs. E. Wickens for writing subroutines used in the analysis, and Messrs M. J. Kellaway and R. L. Nelson for permission to quote examples of applications. The work was carried out at the Central Electricity Research Laboratories, and is published by permission of the Central Electricity Generating Board.

\section{APPENDIX I}

The orthogonality of $[\phi]$

A complex matrix $[\phi]$ is orthogonal if its inverse is equal to its conjugate transpose, that is, if

$$
[\phi]^{-1}=[\phi]^{* T}
$$


The conjugate transpose of $[\phi]$ is $[\theta]$, with elements $\theta_{p i}$ given by equation (12), and if a matrix $[\Lambda]$ is defined as

$$
[\Lambda]=[\phi][\theta]
$$

then $[\theta]=[\phi]^{-1}$ if and only if $[\Lambda]=[\mathscr{I}]$, the unit matrix. The elements of $[\Lambda]$ are $\lambda_{j l}$, and

$$
\lambda_{j l}=M / N \sum_{k=1}^{N / M} \phi_{j k} \theta_{k l}
$$

or

$\lambda_{j l}=M / N \sum_{k=1}^{N / M} \exp [-2 \pi \mathrm{i}(k-1)(L+M(j-1)) / N] \exp [2 \pi \mathrm{i}(k-1)(L+M(l-1)) / N]$.

Now, if $j=l$, equation (50) gives

$$
\lambda_{i j}=M / N \sum_{k=1}^{N / M} \exp (0)=1
$$

If $j \neq l$, equation (50) gives

$$
\lambda_{j l}=M / N \sum_{k=1}^{N / M} \exp [-2 \pi \mathrm{i}(k-1) M / N(j-l)]
$$

Each element in the series defined in equation (52) is a unit vector in the complex plane, rotated through an angle $-2 \pi(j-l)(k-1) M / N$ with respect to the $x$-axis. The angle between successive vectors in the series is $-2 \pi(j-l) M / N$. After $N / M$ terms, the total angle turned through is therefore $-2 \pi(j-l)$. This is an integral multiple of $2 \pi$. The vectors in the series form a closed polygon (see Figure 5 for example) and the vector sum is therefore zero. Thus

$$
\begin{aligned}
& \lambda_{j j}=1 \\
& \lambda_{j l}=0, j \neq l
\end{aligned}
$$

so $\Lambda$ is the unit matrix, $[\theta]=[\phi]^{* \mathrm{~T}}$ is the inverse of $[\phi]$, and $[\phi]$ is orthogonal.

(a) $\mathrm{j}-1=-1$

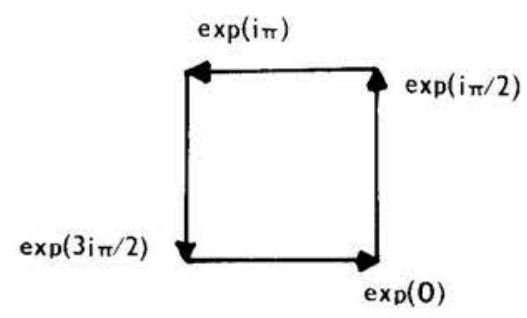

(b) $\mathrm{j}-\mathrm{l}=-2$

$\exp (\mathrm{i} \pi)$

$\exp (0)$

Figure 5. Examples of vector summations of the series in equation (52), with $M=6, N=24$ and $N / M=4$ 


\section{APPENDIX II}

\section{Notation}

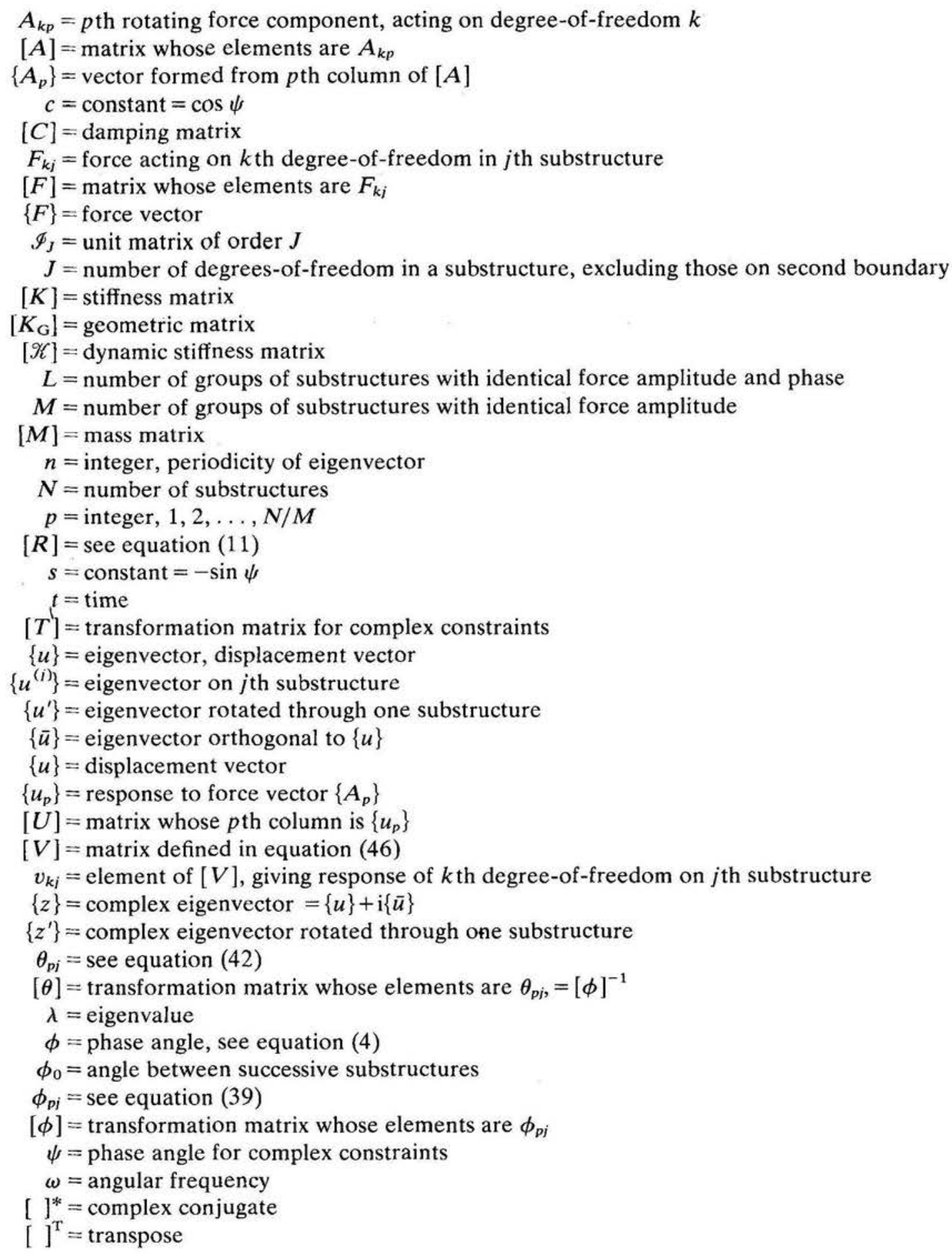




\section{REFERENCES}

1. J. M. Deb Natb, 'Use of higher order displacement functions in the free vibration analysis of shells of revolution having meridional singularities', J. Sound and Vibration, 36, 253-272 (1974).

2. M. G. Hashish and Salman H. Abu-Sitta, 'Response of hyperbolic cooling towers to turbulent wind', J. Struct. Div. ASCE, ST5, 1037-1051 (1974).

3. R. M. Orris and M. Petyt, 'A finite element study of harmonic wave propagation in periodic structures', J. Sound and Vibration, 33, 223-236 (1974).

4. D. L. Thomas, 'Standing waves in rotationally periodic structures', J. Sound and Vibration, 37, 288-290 (1974).

5. R. H. MacNeal, R. L. Harder and J. B. Mason, 'NASTRAN cyclic symmetry capability', NASTRAN Users Experiences 3rd Colloq., Langley Research Centre, Hampton, Virginia, U.S.A., 395-421 (1973), NASA Tech. Memo. NASA TMX-2893.

6. B. Irons, 'Structural eigenvalue problems: elimination of unwanted variables', AIAA J. 3, 961-962 (1965).

7. J. M. Deb Nath, 'Free vibration, stability and 'non-classical modes' of cooling tower shells', J. Sound and Vibration, 33, 79-101 (1974).

8. R. L. Nelson and D. L. Thomas, 'Free vibration analysis of cooling tower with column supports', $J$. Sound and Vibration, 57, 149-153 (1978).

9. P. E. Winney, 'The modal properties of model and full scale cooling towers', J. Sound and Vibration, 57, 131-148 (1978).

10. R. L. Nelson, 'Investigation of shell and axisymmetric shell structures', Ph.D. Thesis, City University, London, 1978. 